\title{
Motivação para mudança de comportamento em amostra de jogadores patológicos
}

\author{
Motivation for behavior change in a sample of pathological gamblers \\ Cristiane Cauduro de Souza', Jaqueline Garcia da Silva', Margareth da Silva Oliveira²
}

\section{Palavras-chave \\ URICA, motivação para mudança, estágios de mudança, jogo patológico.}

\section{RESUMO}

Objetivo: O objetivo deste trabalho foi conhecer o perfil e avaliar as características de jogadores patológicos que frequentavam clínicas e grupos especializados no tratamento desse problema, quanto à motivação para mudança de comportamento. Método: Foram avaliados 69 sujeitos com diagnóstico de jogo patológico, segundo critérios diagnósticos do DSM-IV-TR. A amostra foi subdividida em dois grupos, conforme o tipo de tratamento: grupo em tratamento ambulatorial (TA) e grupo de jogadores anônimos (JA). Os instrumentos utilizados foram a University of Rhode Island Change Assessment (URICA), a Régua de Prontidão e a South Oaks Gambling Screen (SOGS). Resultados: A análise dos resultados evidenciou que o grupo TA apresentou escore médio maior do que o do grupo JA tanto no estágio de pré-contemplação quanto no estágio de ação. Comparando os grupos TA e JA com relação ao tempo de abstinência, observou-se que o grupo de JA está associado a um tempo maior de abstinência do que o grupo de ambulatório. Conclusão: A importância de pesquisar estratégias que favoreçam a compreensão e a adesão aos tratamentos para jogadores patológicos é fundamental. A avaliação da motivação e dos estágios para mudança permite uma direção para o tratamento, auxiliando na elaboração de estratégias terapêuticas.

\begin{abstract}
Objective: The goal of this study was to evaluate the profile and the characteristics of pathological gamblers who attended clinics and groups specialized in the treatment of this problem, focusing on patients' motivation to change this behavior. Method: 69 subjects with a diagnosis of pathological gambling, according to diagnostic criteria of DSM-IV-TR were evaluated. The sample was divided in two groups according to treatment regimen: ambulatory care group and gamblers anonymous group (GA). The instruments used were the University of Rhode Island Change Assessment (URICA), the Ruler of Readiness and SOGS (South Oaks Gambling Screen). Results: The results showed that the ambulatory group had a higher mean score than the GA group, both in the precontemplation stage and in the action stage. Comparing the group of ambulatory care and GA with regard to length of abstinence, it was observed that the group of GA is associated with a longer period of abstinence than the ambulatory group. Conclusion: The importance of sear-
\end{abstract}

Recebido em

$14 / 12 / 2010$

Aprovado em

9/3/2011
1 Pontifícia Universidade Católica do Rio Grande do Sul (PUCRS), Faculdade de Psicologia.

2 Pontifícia Universidade Católica do Rio Grande do Sul (PUCRS), Faculdade de Psicologia, Grupo de Avaliação e Atendimento em Psicoterapia Cognitiva.
Endereço para correspondência: Jaqueline Garcia da Silva Programa de Pós-Graduação em Psicologia Clínica - PUCRS Av. Ipiranga, 6681, Parthenon - 90619-900 - Porto Alegre, RS Telefones: (51) 9981-9403/(51) 9337-3505/(51) 9983-3363/(51) 3320-3500 -ramal: 7749/Telefax: (51) 3320-3500 E-mail: garcia.jake@hotmail.com 


\section{Keywords}

URICA, motivation to change, stages of change, pathological gambling. ching strategies that enhance the understanding and treatment adherence for pathological gamblers is crucial. The evaluation of the motivation to change and the stages of change allow professionals to find a better direction for treatment, assisting in the development of therapeutic strategies.

\section{INTRODUÇÃO}

O Modelo Transteórico de Mudança de Comportamento Prochaska' faz uma contribuição importante no entendimento do processo de motivação que envolve mudança de comportamentos-problema, como o jogo patológico (JP). O JP, por definição, consiste de frequentes e repetidos episódios de jogo que dominam a vida do indivíduo em detrimento de valores e compromissos sociais, ocupacionais, materiais e familiares (CID-10)․ Ele tem sido abordado clinicamente como uma dependência não química.

Esse modelo tem como principal pressuposto que as mudanças bem-sucedidas dependem da aplicação de estratégias certas (processos) na hora certa (estágios)³. O modelo transteórico foca a mudança intencional, ou seja, a tomada de decisão que o indivíduo realiza. Sendo assim, a mudança comportamental acontece ao longo de um processo, no qual as pessoas passam por diversos níveis de motivação para mudança ${ }^{4}$. Esses níveis estariam representados por estágios de motivação para mudança que representam a dimensão temporal do modelo transteórico e permitem que se entenda quando mudanças particulares, intenções e reais comportamentos podem acontecer ${ }^{4}$.

Outro conceito fundamental relacionado ao estudo da motivação é o de prontidão. Segundo DiClemente et al. ${ }^{5}$, existem dois aspectos distintos relacionados à prontidão: prontidão para mudança e prontidão para tratamento. Ainda para esses autores, a prontidão para mudança foi conceituada como a integração entre a conscientização da pessoa sobre seu problema, somada à confiança em suas habilidades para mudar. Já a prontidão para tratamento focaliza a motivação para procurar ajuda, para iniciar, manter e terminar um tratamento. Assim, um jogador patológico pode procurar tratamento e iniciá-lo (alta prontidão para o tratamento), sem estar realmente pronto para permanecer em abstinência do jogo (baixa prontidão para mudar o comportamento-problema).

Sabe-se que uma das variáveis mais importantes implicadas na adesão ao tratamento é a motivação para mudança, ${ }^{6,7}$. Jiménez-Murcia et al. ${ }^{8}$, examinando a efetividade das modalidades terapêuticas grupais entre jogadores patológicos, em um estudo realizado na Espanha, evidenciou que a maior taxa de abandonos e recaídas se produzia nas primeiras cinco semanas de tratamento. Para esses autores, o maior esforço considerando a adesão deveria acontecer no primeiro mês e meio de tratamento e teria de levar em conta aspectos fundamentais, como ser pouco intrusivo no estilo de vida dos pacientes e orientar o trabalho nos fatores de motivação para mudança desse comportamento.

Prochaska et al. ${ }^{3}$ afirmam que avaliar a motivação para mudança, independentemente do tratamento utilizado, parece ser um aspecto importante para a utilização de intervenções adequadas aos pacientes. O resultado de uma correta avaliação implica melhores estratégias para a promoção da motivação para mudança de comportamento.

Castro e Passos ${ }^{9}$, em um trabalho de revisão da literatura, identificaram quatro escalas com parâmetros psicométricos variando de bom a excelente e em condições de serem utilizadas para avaliação da motivação para tratamento e predição de adesão a ele: University Rhode Island Change Assessment (URICA), desenvolvida por McConnaughy et al. ${ }^{10}$, Stages of Change Readiness and Treatment Eargness Scale (SOCRATES), criada por Miller e Tonigan ${ }^{11}$, utilizada especificamente para o comportamento de beber, Readiness to Change Questionnaire (RCQ) e Treatment Motivation Questionnaire (TMQ). Somente as duas primeiras possuem versões validadas para o português.

Callaghan et al. ${ }^{12}$ utilizaram a URICA para avaliar o estágio motivacional de adolescentes usuários de drogas inseridos em um programa de tratamento. O estudo comprovou a validade do Modelo Transteórico considerando que os adolescentes que tinham pontuação elevada na pré-contemplação eram os que apresentavam desistências do programa.

Gómez-Peña et al. ${ }^{13}$ avaliaram a motivação para mudança utilizando a URICA em uma amostra de 531 homens com diagnóstico de jogo patológico, a estrutura interna da escala foi confirmada, assim como uma baixa motivação associada à menor gravidade dos sintomas. Sendo assim, quanto mais grave o sintoma, maior a consciência das consequências dos efeitos negativos da doença e motivação aumentada.

Estudos que avaliaram intervenções como a entrevista motivacional em jogadores patológicos indicam redução de gastos com jogo, redução dos sintomas do transtorno, bem como correlações positivas, quando a intervenção é associada à terapia cognitivo-comportamental ${ }^{14-17}$. Esses achados reforçam a importância de avaliar e trabalhar a motivação de JP.

Outro fator a ser considerado se refere a taxas mais elevadas de JP em alguns grupos como adolescentes, dependen- 
tes químicos, pacientes psiquiátricos, determinados grupos culturais e idosos $^{18}$. Os autores sugerem estudos longitudinais para melhor entendimento da motivação nesses sujeitos. Esses fatores podem ter impacto na motivação desses pacientes, assim como aspectos relacionados à impulsividade e à compulsão ${ }^{19}$.

Em decorrência da utilidade da URICA tanto na área de pesquisa como na área clínica, o objetivo deste trabalho é identificar características do jogador patológico e avaliar a motivação para mudança desse comportamento nesta amostra.

\section{MÉTODO}

Delineamento: Foi realizado um estudo transversal, metodologia quantitativa.

Participantes: Foi selecionada uma amostra de 69 sujeitos que estavam ingressando em tratamento ambulatorial (TA) (entrevista de triagem) ou participando de grupos de jogadores anônimos (JA). Foram convidadas de forma consecutiva todas as pessoas que preenchiam os critérios de inclusão (ser jogador patológico, ter entre 20 e 70 anos, ser do sexo masculino ou feminino e ter, no mínimo, cinco anos de estudo). Os critérios de exclusão foram: transtorno psiquiátrico grave, psicose, risco de suicídio, déficits cognitivos severos ou intoxicação por substâncias psicoativas, indicados pela avaliação realizada na instituição em que o tratamento foi realizado ou pelos dados coletados e informados pelo paciente na entrevista estruturada.

\section{Instrumentos}

- Entrevista estruturada: a coleta de dados sociodemográficos realizou-se mediante entrevista estruturada construída para o estudo.

- Entrevista clínica semiestruturada conforme DSM-IV-TR para avaliar critérios diagnósticos para jogo patológico ${ }^{20}$.

- University of Rhode Island Change Assessment Scale (URICA) (desenvolvida por McConnaughy et al. ${ }^{10}$ 1983): é uma escala de autorrelato que contém 32 itens na versão original e 24 itens na sua versão reduzida, desenvolvida para medir a prontidão para mudança e o estágio motivacional no qual o sujeito se encontra. Constitui-se de quatro subescalas que abrangem os seguintes estágios de mudança comportamental: pré-contemplação (6 itens), contemplação (6 itens), ação (6 itens) e manutenção (6 itens).

- SOGS (South Oaks Gambling Screen): os dados relativos a padrão de jogo e evolução clínica foram obtidos por meio do questionário SOGS. Essa escala foi validada e adaptada para a população brasileira por
Oliveira et al.21, com o objetivo de discriminar jogadores patológicos de jogadores não patológicos, como também diferenciar grupos clínicos de não clínicos e identificar graus distintos de gravidade do hábito de jogar (jogador patológico, jogador social e não tem problemas com o hábito de jogar). A escala original tem 20 itens, e 5 é o ponto de corte para avaliar se a pessoa é um provável jogador patológico.

- Régua de prontidão: é uma técnica analógico-visual, simples e eficaz, para averiguar em qual estágio de mudança o sujeito se encontra. O avaliador pergunta ao sujeito qual dos pontos da régua melhor reflete o quão pronto ele está, no presente momento, para mudar seu comportamento-problema ${ }^{22}$.

\section{Procedimentos}

A pesquisa foi aprovada pelo Comitê de Ética em Pesquisa da PUCRS. Todos os participantes realizaram os procedimentos éticos de pesquisa assinando o termo de consentimento livre e esclarecido e realizaram o preenchimento dos instrumentos orientados pelo pesquisador ou auxiliar de pesquisa previamente treinado. Também foi realizado um estudo piloto para a viabilização deste projeto. A coleta de dados foi realizada em locais de atendimento ambulatorial, no Ambulatório do Jogo Patológico (AMJO) da Universidade de São Paulo (USP), em São Paulo (entrevista de triagem), e no Grupo de Jogadores Anônimos da Cruz Vermelha, de Porto Alegre.

\section{Análise dos dados}

Os dados foram tabulados e as análises estatísticas foram realizadas pelo programa Statistical Package for the Social Sciences (SPSS) versão 17.0. A análise exploratória dos dados foi realizada por meio de medidas descritivas para os dados sociodemográficos: médias, desvio-padrão e frequências. Foram utilizados para análise de comparações de grupo os testes T de Student e qui-quadrado. Para amostras independentes e para a comparação das médias da escala URICA, foi utilizada a análise de variância (ANOVA). O nível de significância utilizado foi de 5\%.

\section{RESULTADOS}

Os participantes deste estudo tinham entre 23 e 68 anos, com média de idade de 44,54 ( $D P=10,15)$. Segundo os critérios de classificação econômica brasileira (IBGE, 2004)23, $50,7 \%$ da amostra encontraram-se predominantemente na classe média. A renda mensal familiar variou de 1 a 27 salários-mínimos, $R \$ 320,00$ até $R \$ 14.000,00$. Todos os participantes possuem diagnóstico de jogo patológico, segundo a entrevista estruturada baseada nos critérios do DSM-IV-TR' ${ }^{15}$, 
e foram coletados em locais de atendimento ambulatorial $(60,9 \%)$ e em grupos de jogadores anônimos (39,1\%). A descrição detalhada das variáveis sociodemográficas da amostra encontra-se na tabela 1.

Tabela 1. Distribuição da amostra, segundo dados sociodemográficos $(n=69)$

\begin{tabular}{lll}
\hline Dados sociodemográficos & $\%$ & $\mathbf{n}$ \\
\hline Sexo & 52,2 & 36 \\
Masculino & 47,8 & 33 \\
Feminino & & \\
Escolaridade & 14,5 & 10 \\
5 a 8 anos & 42,0 & 29 \\
9 a 11 anos & 43,5 & 30 \\
Acima de 12 anos & & \\
Situação trabalho & 76,8 & 53 \\
Trabalha & 23,2 & 16 \\
Não trabalha & & \\
Nível socioeconômico & 2,9 & 2 \\
Classe A & 20,3 & 14 \\
Classe B & 50,7 & 35 \\
Classe C & 23,2 & 16 \\
Classe D & 2,90 & 2 \\
Classe E & & \\
Estado civil & 31,9 & 22 \\
Solteiro & 53,6 & 37 \\
Casado & 13,0 & 9 \\
Separado & 1,5 & 1 \\
Viúvo & & \\
Regime de tratamento & 60,9 & 27 \\
Ambulatório & 39,1 & \\
JA & & \\
\hline
\end{tabular}

\section{Histórico clínico}

Da amostra estudada, a partir da entrevista semiestruturada, $34,8 \%$ relataram que fazem uso de álcool e/ou tabaco. Ainda, com relação ao uso de drogas e álcool, 34,8\% revelaram ter tido pai ou mãe com problemas com relação ao uso de álcool ou drogas ao longo da vida. Do total da amostra, é importante salientar que $29,2 \%$ disseram que estão fazendo uso de medicação psiquiátrica atualmente, tendo recebido diagnóstico de depressão ou ansiedade por profissionais médicos.

\section{Resultados da SOGS - Características do padrão de jogo}

Do total da amostra, 97,1\% reconhecem ter problemas com o hábito de jogar. Segundo os resultados da SOGS, os 98,6\% dos sujeitos entrevistados foram classificados como prováveis jogadores patológicos. A média de pontos na escala SOGS foi de 13,36 pontos e DP = 3,32, e os escores variaram entre 4 e 19 pontos.

Quanto à abstinência, 57,1\% tinham interrompido as apostas em jogos há menos de 30 dias, 22,9\% não estavam jogando no período de 30 a 180 dias e somente 18,6\% não jogavam há mais de seis meses.
Quando perguntados sobre a maior quantia de dinheiro utilizada no jogo em um único dia, 56,5\% da amostra disseram ter gasto entre $R \$ 250,00$ e $R \$ 2.500,00$ e 30,4\% disseram ter gasto mais de $\mathrm{R} \$ 2.500,00 \mathrm{em}$ um dia de jogo. Com relação aos pais já terem tido problemas com jogo, 20,3\% dos sujeitos disseram que o pai joga (ou jogava) demais e 10,1\% dos sujeitos disseram que tanto mãe quanto o pai tinham o hábito de jogar.

Com relação ao hábito de jogar, a média de idade de início do jogo com apostas em loterias (sena, jogo do bicho, loto, entre outros) é de 22,31 anos ( $D P=9,43$ ); já a média de idade de início do jogo de bingo é de $31,11(\mathrm{DP}=11,03)$ e do videopôquer é de 32,89 anos ( $\mathrm{DP}=12,38$ ). Na amostra estudada, os tipos de jogos de preferência relatados pelos sujeitos são: videopôquer ou outras máquinas de jogos (82,6\%), bingo (79,7\%), loterias (sena, jogo do bicho, loto) $(88,4 \%)$ e jogo de cartas a dinheiro (56,5\%). A figura 1 apresenta a distribuição da amostra com relação aos tipos de jogos praticados na vida.

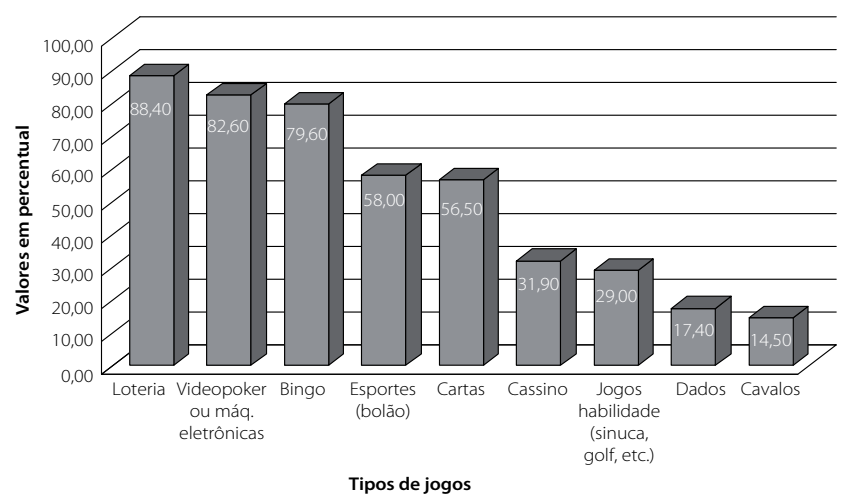

Figura 1. Distribuição da amostra quanto aos tipos de jogos praticados na vida.

\section{Estágios motivacionais, prontidão para mudança e tipo de tratamento}

A amostra foi subdividida em dois grupos, conforme o regime de tratamento TA e o grupo de JA. Utilizando-se o teste $T$, verificaram-se diferenças significativas entre os grupos com relação à motivação para mudança no estágio de pré-contemplação $(p=0,005)$ e no estágio de manutenção $(p=0$ 044). Na tabela 2, pode-se observar que no TA o escore médio foi significativamente maior do que no grupo JA tanto no estágio de pré-contemplação quanto no estágio de manutenção.

\section{Regime de tratamento e período de abstinência}

Na tabela 3, pode-se observar que, segundo o teste qui-quadrado, o grupo TA está associado ao período de tempo de abstinência de 30 a 180 dias, enquanto o JA está significativamente associado a um tempo de abstinência superior a 180 dias $(p=0,04)$, indicando que, nesta amostra, o grupo de JA manteve-se por um período maior de tempo em abstinência. 
Tabela 2. Comparação entre o grupo TA e o grupo JA quanto à motivação para mudança

\begin{tabular}{|c|c|c|c|c|c|c|}
\hline & & & & & & \\
\hline Estágios da URICA & Média & DP & Média & DP & $\mathrm{t}$ & $\mathrm{p}$ \\
\hline Pré-contemplação & 15,5 & 3,63 & 12,29 & 5,62 & 2,876 & 0,005 \\
\hline Contemplação & 21,80 & 3,41 & 22,22 & 2,48 & $-0,542$ & 0,590 \\
\hline Ação & 24,92 & 4,95 & 25,74 & 3,87 & 2,049 & 0,044 \\
\hline Manutenção & 25,09 & 4,83 & 22,44 & 5,82 & $-0,721$ & 0474 \\
\hline Prontidão & 10,11 & 2,17 & 10,42 & 1,82 & $-0,615$ & 0,541 \\
\hline
\end{tabular}

*Teste T de Student - nível de significância 5\%.

Tabela 3. Distribuição da amostra em relação ao regime de tratamento e o período de abstinência

\begin{tabular}{|c|c|c|c|}
\hline \multirow{2}{*}{ Tempo de abstinência } & \multicolumn{2}{|c|}{ Regime de Tratamento } & \multirow{2}{*}{ Total } \\
\hline & TA & JA & \\
\hline \multicolumn{4}{|l|}{ Até 30 dias } \\
\hline $\mathrm{n}$ & 26 & 14 & 40 \\
\hline$\%$ & 61,9 & 51,9 & 58 \\
\hline \multicolumn{4}{|l|}{30 a 180 dias } \\
\hline $\mathrm{n}$ & 13 & 3 & 16 \\
\hline$\%$ & 31 & 11,1 & 23,2 \\
\hline \multicolumn{4}{|l|}{ Mais de 180 dias } \\
\hline $\mathrm{n}$ & 3 & 10 & 13 \\
\hline$\%$ & 7,1 & 37 & 18,8 \\
\hline Total & 42 & 27 & 69 \\
\hline
\end{tabular}

${ }^{*}$ Teste Qui-Quadrado:10,872(a)df $=2(p=0,04)$, nível de significância 5\%.

\section{Estágios de motivação, prontidão para mudança e período de abstinência}

Foi realizada uma análise de variância (ANOVA) e observou-se que não existe diferença significativa entre o tempo de abstinência e os estágios motivacionais. Os resultados estão descritos na tabela 4.

Tabela 4. Distribuição das médias e desvios-padrão dos estágios motivacionais, prontidão em relação ao período de abstinência

\begin{tabular}{|c|c|c|c|c|c|}
\hline $\begin{array}{l}\text { Estágios da URICA/tempo de } \\
\text { abstinência }\end{array}$ & $\mathrm{n}$ & Média & DP & $\mathbf{F}$ & $\mathbf{p}^{*}$ \\
\hline \multicolumn{6}{|l|}{ Pré-contemplação } \\
\hline Até 30 dias & 40 & 14,35 & 5,33 & \multirow{4}{*}{0,565} & \multirow{4}{*}{0,571} \\
\hline 30 a 180 dias & 16 & 14,93 & 2,93 & & \\
\hline Mais de 180 dias & 13 & 13,07 & 4,73 & & \\
\hline Total & 69 & 14,24 & 4,75 & & \\
\hline \multicolumn{6}{|l|}{ Contemplação } \\
\hline Até 30 dias & 40 & 22,40 & 3,06 & \multirow{4}{*}{1,051} & \multirow{4}{*}{0,355} \\
\hline 30 a 180 dias & 16 & 21,12 & 3,50 & & \\
\hline Mais de 180 dias & 13 & 21,69 & 2,46 & & \\
\hline Total & 69 & 21,97 & 3,07 & & \\
\hline \multicolumn{6}{|l|}{ Ação } \\
\hline Até 30 dias & 40 & 25,57 & 4,66 & \multirow{4}{*}{0,279} & \multirow{4}{*}{0,758} \\
\hline 30 a 180 dias & 16 & 25,00 & 4,61 & & \\
\hline Mais de 180 dias & 13 & 24,53 & 4,35 & & \\
\hline Total & 69 & 25,24 & 4,55 & & \\
\hline \multicolumn{6}{|l|}{ Manutenção } \\
\hline Até 30 dias & 40 & 24,57 & 4,88 & \multirow{4}{*}{1,103} & \multirow{4}{*}{0,338} \\
\hline 30 a 180 dias & 16 & 24,37 & 5,54 & & \\
\hline Mais de 180 dias & 13 & 22,07 & 6,47 & & \\
\hline Total & 69 & 24,05 & 5,36 & & \\
\hline \multicolumn{6}{|l|}{ Prontidão } \\
\hline Até 30 dias & 40 & 10,44 & 2,01 & \multirow{4}{*}{0,497} & \multirow{4}{*}{0,611} \\
\hline 30 a 180 dias & 16 & 9,96 & 2,26 & & \\
\hline Mais de 180 dias & 13 & 9,92 & 1,88 & & \\
\hline Total & 69 & 10,23 & 2,03 & & \\
\hline
\end{tabular}

* Análise de variância (ANOVA) - nível de significância 5\%.

\section{DISCUSSÃO}

A amostra foi subdividida em dois grupos, conforme o regime de tratamento: pacientes com TA e pessoas que frequentavam grupos de JA.

O grupo TA apresentou escore médio maior do que o grupo JA tanto no estágio de pré-contemplação quanto no estágio de ação. Pode-se inferir que os pacientes que buscaram atendimento ambulatorial, por terem sido avaliados na entrevista de triagem, poderiam estar procurando atendimento mais pelas consequências negativas da gravidade do jogo, que perturbam a família e interferem no trabalho, na vida financeira e nas relações sociais, do que de fato pela motivação intrínseca para modificar seu comportamento com vistas à abstinência do comportamento de apostar em jogos de azar. Por outro lado, deve-se lembrar que essa é uma escala de autorrelato, com medidas subjetivas, e isso pode ser uma limitação do instrumento. O fato de esse grupo também pontuar mais no estágio de ação pode nos fazer refletir que a simples procura pelo atendimento, que é fundamental, não quer dizer que a pessoa esteja motivada para modificar seu comportamento com relação ao jogo, ou que está preparada para ficar em abstinência.

Comparando o grupo TA e o grupo de JA com relação ao tempo de abstinência, observou-se que o grupo de JA está associado há um tempo maior de abstinência do que o grupo de ambulatório. Cabe ressaltar que o JA trabalha com os 12 passos de recuperação, filosofia reconhecida mundialmente e utilizada pelos alcoólicos anônimos (AA), e que ele tem íntima relação com os processos que envolvem a motivação, reforçando a ideia de que o trabalho terapêutico que foca a motivação do paciente pode ser muito útil. Alguns autores acreditam que a participação em organizações do tipo "jogadores anônimos" é benéfica e a mais efetiva opção de tratamento, por combater a negação e as projeções, demandando honestidade e responsabilidade (Custer, 1984) ${ }^{24}$. Um estudo de seguimento realizado entre jogadores patológicos previamente tratados antes do encaminhamento ao JA concluiu que o índice de adesão ao JA e a abstinência são significativamente maiores entre os pacientes submetidos previamente a outras intervenções ou propostas de reabilitação ${ }^{25}$.

A comparação dos grupos em diferentes momentos segue exemplos como o estudo de Oei et al. ${ }^{17}$, que comparou indivíduos submetidos a intervenção com terapia cognitivo-comportamental e entrevista motivacional com um grupo 
em lista de espera. Ao mesmo tempo que os sujeitos estão em diferentes momentos e pode ser um viés, torna-se possível conhecer aspectos distintos da mesma população.

Autores discutem fatores como a possibilidade de que, quando uma pessoa percebe que tem um problema grave que requer tratamento profissional, ela pode decidir mudar o comportamento e, por vezes, fazê-lo por si própria. Além disso, existe o fato de que uma entrevista de avaliação pode ser o necessário para uma pessoa parar de jogar. Quando um tratamento ativo é comparado com controles, a literatura frequentemente mostra que o controle sem tratamento pode ser bastante eficaz, pelo menos no curto prazo ${ }^{26}$.

DiClemente et al. ${ }^{5}$ afirmaram que a avaliação do nível de prontidão para mudança, como uma medida mais dinâmica e abrangente do que a ideia de estágios motivacionais, pode ser muito útil. Eles avaliaram a prontidão motivacional de pacientes ambulatoriais em início de tratamento para o alcoolismo e produziram três grupos: um grupo de baixa prontidão, com pontuação média de 8,7, um grupo de média prontidão, com média de 10,5, e um grupo com alta prontidão, com média de 12,4. Esses grupos estão em paralelo com os perfis da pré-contemplação, contemplação e preparação descritos anteriormente.

O perfil do jogador apresentado neste estudo é semeIhante ao de estudos anteriores, como o de Oliveira ${ }^{27}$, no qual chamam a atenção a escolaridade e o nível socioeconômico elevado, se comparados a amostras de usuários de drogas. Nesta amostra, o grau de escolaridade elevado, a renda mensal e o estado civil (a maioria constituiu família) podem sugerir que o processo de instalação da dependência do hábito de jogar ocorre de forma silenciosa e em ritmo diferente das demais dependências.

Outro dado importante é a idade de início do hábito de jogar, que apresentou médias distintas para cada tipo de jogo, o que poderia ser explicado também pelo critério de acessibilidade (lotéricas e máquinas eletrônicas estão em lugares públicos de fácil acesso), indicando que o início dessa prática na amostra pesquisada se dá no começo da idade adulta e que pode se tornar um problema na vida do indivíduo, em período de tempo razoavelmente curto. Cabe ressaltar que a amostra estudada não incluiu uma faixa etária mais jovem (adolescentes), por entender que ela apresenta particularidades com relação ao hábito de jogar envolvendo principalmente a internet e jogos eletrônicos. Mas reconhecemos a necessidade de investigação nessa área, já que há evidências de que a dependência do jogo entre os jovens é um dado alarmante.

\section{CONCLUSÃO}

De maneira geral, o jogo patológico parece ser uma condição tratável e o prognóstico, segundo resultados de programas de tratamento descritos neste trabalho, pode depender das diferentes técnicas e abordagens do tratamento e da motivação do sujeito. Os dados obtidos neste estudo, considerando o perfil da amostra encontrada, os problemas decorrentes do hábito de jogar, bem como o estágio motivacional em que os pacientes se encontram, fornecem indicativos de comportamentos dessa população. A partir dos achados, é reforçada a importância de pesquisar fatores que favoreçam a compreensão e a adesão aos tratamentos para jogadores patológicos. A avaliação da motivação e dos estágios para mudança permite uma direção para o tratamento e contribui de forma relevante para o planejamento e a elaboração de estratégias terapêuticas.

Cabe ressaltar que o objetivo inicial deste estudo era conhecer o perfil da amostra em questão e que a grande dificuldade do trabalho foi sem dúvida acessar os jogadores. Desde a proibição dos jogos de azar no Brasil, parece ter havido uma diminuição da procura pelo atendimento, conforme o AMJO (USP-SP) e o grupo de JA da Cruz Vermelha de Porto Alegre. Portanto, diante da escassez de pacientes, não foi possível controlar as variáveis tempo de tratamento e tipo de tratamento, o que favoreceria a comparação entre os grupos de maneira controlada. No entanto, os resultados apontaram para aspectos importantes, e a diferença entre os grupos possibilitou o conhecimento das características que podem ser encontradas em jogadores em distintos momentos e abordagens de tratamento.

\section{CONFLITOS DE INTERESSE}

Os autores declaram não haver quaisquer conflitos de interesse.

\section{REFERÊNCIAS}

1. Prochaska J0. Systems of psychotherapy: a transtheorical analysis. Homewood, Illinois: Dorsey Press; 1979

2. Organização Mundial da Saúde. Classificação de transtornos mentais e de comportamento da CID-10: referência rápida. Porto Alegre: Artes Médicas; 1997.

3. Prochaska J0, DiClemente CC, Norcross JC. In search of how people change: applications to addictive behaviour. Am Psychol. 1992;47:102-14.

4. Szupszynski KPDR, Oliveira MS. Adaptação brasileira da University Rohde Island Change Assessment (URICA) para usuários de substâncias ilícitas. PsicoUsf. 2008;13(1):31-9.

5. DiClemente CC, Schlundt BS, Gemmell L. Readiness and stages of change in addiction treatment. Am J Addict. 2004;13:130-19.

6. Hodgins DC. Implications of a brief intervention trial for problem gambling for future outcome research. J Gambl Stud. 2005;21:13-9.

7. Toneatto $\mathrm{T}$, Millar $\mathrm{G}$. The assessment in treatment of problem gambling: a critical review of the literature. Psychol Addict Behav. 2004;17:284-92.

8. Jiménez-Murcia S, Alvarez EM, Granero RP, Aymani MN, Gómez MP, Jaurrieta N, et al. Análisis de la eficacia de dos modalidades de tratamiento cognitivo conductual grupal para el juego patológico. Psicol Conduct. 2005;13(3):495-510.

9. Castro M, Passos S. Entrevista motivacional e escalas de motivação para tratamento em dependência de drogas. Rev Bras Psiquiatr. 2005;32(6):330-5.

10. McConnaughy EA, Prochaska J0, Velicer WE. Stages of change in psychotherapy: measurement and sample profiles. Psychother Theor Res Pract. 1983;20:368-75. 
11. Miller WR, Tonigan JS. The Stages of Change Readiness and Treatment Eagerness Scale (SOCRATES). Psychol Addict Behav. 1996;10:81-9.

12. Callaghan RC, Hathaway A, Cunningham JA, Vettese LC, WyattS, Taylor L. Does stage-of-change predict dropout in a culturally diverse sample of adolescents admitted to inpatient substance-abuse treatment? A test of the Transtheoretical Model. Addict Behav. 2005;30(9):1834-47.

13. Gómez-Peña M, Penelo E, Granero R, Fernández-Aranda F, Alvarez-Moya E, Santamaría JJ, et al. Motivation to change and pathological gambling: analysis of the relationship with clinical and psychopathological variables. Br J Clin Psychol. 2010.

14. Diskin KM, Hodgins DC. A randomized controlled trial of a single session motivational intervention for concerned gamblers. Behav Res Ther. 2009;47:382-8.

15. Grant JE, Donahue CB, Odlaug BL, Kim SW, Miller MJ, Petry NM. Imaginal desensitisation plus motivational interviewing for pathological gambling: randomized controlled trial. $\mathrm{Br}$ J Psychiatry. 2009;195:266-7.

16. Carlbring P, Jonsson J, Josephson H, Forsberg L. Motivational interviewing versus cognitive behavioral group therapy in the treatment of problem and pathological gambling: a randomized controlled trial. Cogn Behav Ther. 2009;34(3):1-12.

17. Oei TPS, Raylu N, Casey LM. Effectiveness of group and individual formats of a combined motivational interviewing and cognitive behavioral treatment program for problem gambling: a randomized controlled trial. Behav Cogn Psychother. 2010;38:233-8.

18. Raylu N, Oei TPS. Pathological gambling: a comprehensive review. Clin Psychol Rev 2002;22(7):1009-61.
19. Blanco C, Potenza MN, Kim SW, Ibáñez A, Zaninelli R, Saiz-Ruiz J, et al. A pilot study of impulsivity and compulsivity in pathological gambling. Psychiatry Res. 2009;167(12):161-8.

20. American Psychiatric Association (APA). Manual Diagnóstico e Estatístico de Transtornos Mentais - DSM-IV-TR (4a ed.) Porto Alegre: Artmed; 2002.

21. Oliveira MP, Silva MT, Silveira DX. Validity study of the South Oaks Gambling Screen (SOGS) among distinct groups of Brazilian Gamblers. Rev Bras Psiquiatr. 2002;24:170-6.

22. Velásquez M, Maurer G, Crouch C, Diclemente C. Group treatment for substance abuse: a stages-of-change therapy manual. New York: The Guilford Press; 2001.

23. IBGE - Instituto Brasileiro de Geografia e Estatística. Perfil dos municípios brasileiros: finanças públicas. Rio de Janeiro: IBGE; 2004. 133p

24. Custer RL. Profile of the pathological gambler. J Clin Psychiatry. 1984;45:35-8.

25. Taber II, McCormick RA, Russo AM. Follow-up of pathological gamblers after treatment. J Clin Psychiatry. 1987;144:757-61.

26. Carlbring P, Jonsson J, Josephson H, Forsberg L. Motivational interviewing versus cognitive behavioral group therapy in the treatment of problem and pathological gambling: a randomized controlled trial. Cogn Behav Ther. 2010;39(2):92-103.

27. Oliveira MPMT. Jogo patológico: um estudo sobre jogadores de bingo, videopôquer e jockey club [dissertação]. São Paulo: Instituto de Psicologia da Universidade de São Paulo; 1997. 